\title{
The Role of the Flash Visual Evoked Potential in Evaluating Visual Function in Patients with Indirect Traumatic Optic Neuropathy
}

This article was published in the following Dove Press journal:

Clinical Ophthalmology

\section{Mai A Mohammed \\ Ehab Mossallam (D) \\ Ibrahim Y Allam}

Ophthalmology Department, Faculty of Medicine, Alexandria University, Alexandria, Egypt
Correspondence: Mai A Mohammed

36 Kamaleldin Salah, Semouha,

Alexandria, Egypt

Tel +201221081590

Fax +2034244441

Email mai.abdalnabi@yahoo.com
Purpose: To assess the value of the flash visual evoked potential (FVEP) in determining final visual prognosis in patients with indirect traumatic optic neuropathy (TON).

Subjects and Methods: We included 30 patients diagnosed with indirect TON. Within one week of the onset of the trauma, visual acuity was recorded, pupillary reactions were assessed, FVEP was performed in both eyes. The amplitudes (N1p1 and N2P2) and the latency of $\mathrm{P} 2$ for each eye were recorded and amplitude ratio of N2P2 between the affected and normal eye was calculated. In follow-up visits, the cases underwent a complete ophthalmic examination, assessment of visual acuity, pupillary reaction, and FVEP.

Results: The study included 22 males $(73.3 \%)$ and 8 females $(26.7 \%)$. The right eye was involved in 16 patients (53.3\%) and left eye was involved in 14 cases (46.7\%). According to the findings of FVEP, there was a direct correlation between final visual acuity and initial amplitude of N1p1 and N2P2 and negative correlation with latency of P2 wave. In 20 patients in whom the N1P1 and N2P2 amplitude was within the normal range and amplitude ratio of N2P2 of normal and fellow eye was at least 0.5 and the P2 implicit time was less than $140 \mathrm{~ms}$, they achieved better visual outcome and visual acuity improved in the affected eye. In other 10 patients in whom the N1P1 and N2P2 amplitude was below normal range and the N2P2 amplitude ratio between the normal and the affected eye was less than 0.5 and the P2 implicit time was more than $140 \mathrm{~ms}$, the visual acuity in the affected eye was less than 0.01 and these patients achieved less or no improvement in their visual function.

Conclusion: Cases with TON usually present with severe loss of vision. FVEP is highly predictive of final visual outcome in patients having indirect TON given that the other eye is normal to be used as the patients' internal control. More studies are needed to confirm these results.

Keywords: flash visual evoked potential; FVEP, orbital computed tomography, traumatic optic neuropathy; TON, International Society of Electrophysiology of Vision; ISCEV

\section{Introduction}

Traumatic optic neuropathy (TON) represents a critical acute injury for the optic nerve that disrupts the visual function and may lead to permanent disability. ${ }^{1,2}$ It was showed that the commonest sites of nerve injury are at the foramina of the optic canal, under the falciform dural fold and the optic nerve canalicular segment. ${ }^{3}$ This results from both direct and indirect trauma. ${ }^{4}$ In direct trauma, stress is usually directed to the optic nerve and is associated with orbital fracture as fragments lacerate optic nerve or cause mechanical contusion. ${ }^{5}$ Moreover, indirect TON is the 
most common type of TON, and its clinical diagnosis is based on evidence of the dysfunction the optic nerve in patients who have craniofacial trauma. Mostly, the examination is normal in the early phases except for relative afferent pupillary defect (RAPD). Hence, performing an orbital computed tomography (CT) scan in these cases is important to detect acute orbital haemorrhages, bony fractures, or fractures to the optic canal. ${ }^{6}$

The mechanism of indirect TON is that the stress is transferred through the oculofacial tissues and skeleton. The coup countercoup forces damage of the nerve at sites between fixed and mobile segments. This leads to compressing and disrupting of pial vessels within the canal which limit the vascular supply of the optic nerve.,

Walsh proposed the concept of primary and secondary injury in TON. ${ }^{8}$ It was shown that the primary injury occurs due to irreversible contusion and necrosis from shearing of retinal ganglion cell axons. Secondary injury occurs due to destructive post injury biochemical and metabolic changes, resulting in optic nerve edema that aggregates the ischemia. 7,9

The main clinical presentation of TON is the loss of the visual function leading to decreased best corrected visual acuity, abnormal color vision or loss of visual field, and presence of RAPD in the cases with unilateral TON. Initial fundus examination may be of value in detecting patients with anterior optic nerve injury who presented with swelling in the optic nerve head and adjacent retinal hemorrhages, but in cases having posterior injuries which are more common, a normal fundus is usually seen. ${ }^{10}$

If the diagnosis of TON is set, the main issue will be the prediction for the degree of visual function and prognosis for vision. In most of the cases, visual acuity is decreased severely. Electrophysiological studies have been used to predict visual outcome after ocular injuries. A variety of methods such as flash electroretinography (ERG), the visual-evoked potential (VEP), and pattern ERG have been used for this purpose.

VEP can measure the cortical activity in the visual system as result to flash or pattern stimulus which can be abnormal in the different types of optic neuropathy. Visual recovery may not be achieved when VEP results cannot be recorded, while a flash VEP amplitude ratio for affected side to normal side $>0.5$ sounds to be predictive of a good visual outcome according to some published studies. ${ }^{11,12}$ There is a clear wider variability in normal ranges for flash VEPs than for pattern VEPs and an inter-ocular comparison is usually the most useful. Although pattern VEPs could be normal, flash VEPs may show abnormalities as documented in some cases of optic neuritis, pathology of optic nerve sheath or unsuspected retinopathy. ${ }^{13-15}$ The purpose of this study was to assess the value of the flash visual evoked potential (FVEP) in evaluating visual function in patients having indirect TON.

\section{Subjects and Methods}

The study was conducted on 30 eyes (30 patients) diagnosed as acute unilateral indirect TON from severe blunt trauma that were attending outpatients clinic of the ophthalmology department of Alexandria Main University Hospital between January 2020 and June 2020. The study included 22 males (73.3\%) and 8 females $(26.7 \%)$. The right eye was involved in 16 patients $(53.3 \%)$ and the left eye was involved in 14 cases $(46.7 \%)$. The fellow eye of 30 patients was clinically free. The study adhered to the tenets of the declaration of Helsinki and Institutional Review Board (the ethical committee of Alexandria University Faculty of Medicine) approval was obtained (IRB NO. 00012098 and serial number: 0304629). Written Informed consent was obtained from the patients. All study participants were subjected to a standard protocol of office examination to exclude any ocular diseases in the affected and the fellow eye of the participants, assessment of visual acuity, RAPD detection, fundus examination, orbital CT to exclude orbital fractures and direct causes of optic neuropathy and scheduled for electrophysiological testing (flash VEP response because some patients had poor fixation and low visual acuity in the affected eye so pattern VEP could not be done) in both eyes. Flash VEP was conducted using the Roland Consult electrophysiology and imaging using the standard protocol of FVEP provided by the International Society of Electrophysiology of Vision (ISCEV) recording P2 implicit time, N1P1 amplitude and N2P2 amplitude. ${ }^{11}$ Both eyes were examined to use the patients normal fellow eye as patients' internal control due to wide variability in normative data of flash VEP and to calculate N2P2 amplitude ratio of two eyes. The electrodes were placed according to the ISCEV standards (reference electrode $(\mathrm{Fz})$ placed at the forehead, ground electrode $(\mathrm{Cz})$ at the vertex, active electrode $(\mathrm{Oz})$ at approximately $2 \mathrm{~cm}$ above the inion (occipital protuberance). All patients were lightadapted (photopic conditions) and the pupils were not dilated. The white flash stimulus (at a rate of $1-2 \mathrm{~Hz}$, strength of 3 photopic cd.s.m ${ }^{-2}$, subtending a visual field of at least $20^{\circ}$ and at a distance of $30 \mathrm{~cm}$ ) was delivered 
using a ganzfeld stimulator (with an impedance of $<10$ $\mathrm{k} \Omega$ ). Follow-up visits at one month and three months, in every visit the patients underwent a thorough ophthalmic examination, assessment of visual acuity and FVEP in the affected eye.

Data were analyzed using SPSS software version 20.0. Numerical data were described using number and percent. Categorical data were described using range (minimum and maximum), mean, standard deviation and median. Paired $t$-test was assessed for normally distributed quantitative variables, to compare between two periods. We used Pearson coefficient to correlate between two normally distributed quantitative variables. ANOVA with repeated measures was assessed for normally distributed quantitative variables, to compare between more than two periods, and Post Hoc test (Bonferroni adjusted) for pairwise comparisons while Friedman test was assessed for abnormally distributed quantitative variables, to compare between more than two periods and Post Hoc Test (Dunn's) for pairwise comparisons. Significance of the obtained results was judged at the $5 \%$ level.

\section{Results}

The study was conducted on 30 eyes (30 patients) diagnosed as acute unilateral indirect TON from severe blunt trauma. Different parameters of the patients are described in Table 1. The mean age of the included patients was 27.8 \pm 8.7 years. The most common cause was motor accidents (cars, bike and other motor accidents). All patients were examined within one to seven days from the insult. CT revealed no evidence of direct TON, there was no fracture of orbital wall in $60 \%$ of the studied group, $40 \%$ had orbital wall fracture with no evidence of optic nerve compression. Patients received no specific treatment and were followed up for three months.

At the time of the presentation, the range of the visual acuity of the studied eyes was light perception in 5 patients to $6 / 60(0-0.1)$ and the clinical examination of the affected eyes was clinically free except for RAPD. After one month, the mean visual acuity was $0.1 \pm 0.1$ and no cases showed drop in their visual acuity with $66.7 \%$ showing improvement and $33.3 \%$ remaining the same. There was statistically significant improvement of visual acuity at the end of the first month $(\mathrm{p}=0.005)$. Those with poor initial visual acuity (light perception or hand motion) show no or little improvement. After three months, the mean visual acuity was $0.2 \pm 0.2$ with $33.3 \%$ of the studied cases showing no improvement and $66.7 \%$ showing
Table I Distribution of the Studied Cases According to Different Parameters (Age, Sex, Eye, Nature of Trauma, Time of Presentation, PARD Relative Defect, and CT Scan Findings) at Baseline $(n=30)$

\begin{tabular}{|l|c|}
\hline & No. (\%) \\
\hline Sex & \\
Male & $22(73.3 \%)$ \\
\hline Age (years) & $8(26.7 \%)$ \\
Mean \pm SD. & \\
Median (Min. - Max.) & $27.8 \pm 8.7$ \\
\hline Eye & $27(18-45)$ \\
Right & \\
Left & $16(53.3 \%)$ \\
\hline Nature of trauma & $14(46.7 \%)$ \\
Car accident & \\
Motor accident & $8(26.7 \%)$ \\
Fall from bike & $6(20 \%)$ \\
Assault & $2(6.7 \%)$ \\
Fall from height & $10(33.3 \%)$ \\
\hline Time of presentation (days) & $4(13.3 \%)$ \\
Mean \pm SD. & \\
Median (Min. - Max.) & $2.7 \pm 1.7$ \\
RAPD relative defect & $2(1-7)$ \\
\hline CT scan finding & $30(100 \%)$ \\
No fracture & $12(40 \%)$ \\
Fracture & $2(16.7 \%)$ \\
Zygomatic fracture & $2(16.7 \%)$ \\
Medial orbital wall fracture & $8(66.7 \%)$ \\
\hline
\end{tabular}

improvement, those cases that show initial improvement continued to improve over a period of three months. There was statistically significant improvement of the visual acuity at the end of third month compared to initial presentation $(p<0.001)$ but there were statistically insignificant changes from the first month to the end of the third month $(\mathrm{p}=0.889)$. We found that the better the initial visual acuity, the more significant improvement over follow-up period. The visual acuity changes were described in Table 2 and 3$)$.

The FVEP findings performed in both eyes of the studied group at the time of the presentation are presented in Table 4. There was statistically significant differences in P2 implicit time, N1-P1 and N2-P2 amplitudes between the affected and the normal fellow eye that's used as the patients' internal control $(\mathrm{p}<0.001)$. In the follow-up visit 
Table 2 Visual Acuity of the Studied Cases at Baseline, One Month, and Three Months $(n=30)$

\begin{tabular}{|c|c|c|c|c|c|}
\hline \multirow[t]{2}{*}{ VA } & \multirow[t]{2}{*}{ Initial } & \multicolumn{2}{|c|}{ Follow-Up } & \multirow[t]{2}{*}{$\mathbf{F r}$} & \multirow[t]{2}{*}{$\mathbf{p}$} \\
\hline & & One Month & Three Months & & \\
\hline Mean \pm SD. & $0 \pm 0$ & $0.1 \pm 0.1$ & $0.2 \pm 0.2$ & \multirow[t]{2}{*}{$20.0^{*}$} & \multirow[t]{2}{*}{$<0.001 *$} \\
\hline Median (Min. - Max.) & $0(0-0.1)$ & $0.1(0-0.3)$ & $0.3(0-0.5)$ & & \\
\hline Sig.bet.periods & \multicolumn{3}{|c|}{$\mathrm{P}_{1}=0.005^{*}, \mathrm{P}_{2}<0.00 \mathrm{I}^{*}, \mathrm{P}_{3}=0.889$} & & \\
\hline
\end{tabular}

Notes: Fr: Friedman test, Sig. bet. periods was done using post hoc test (Dunn's). $\mathrm{p}$ : $\mathrm{p}$ value for comparing between the three periods. $\mathrm{p}_{\mathrm{I}}$ : $\mathrm{p}$ value for comparing between initial and one month. $\mathrm{P}_{2}: \mathrm{p}$ value for comparing between initial and three months. $\mathrm{P}_{3}: \mathrm{p}$ value for comparing between one month and three months. $* S$ tatistically significant at $\mathrm{p} \leq 0.05$.

Table 3 Descriptive Analysis of the Studied Cases According to Improvement of Each Period for Visual Acuity

\begin{tabular}{|l|c|}
\hline Follow-Up & VA Initial \\
\hline One month & \\
Decreased & $0(0 \%)$ \\
No change & $10(33.3 \%)$ \\
Improved & $20(66.7 \%)$ \\
\hline Three months & \\
Decreased & $0(0 \%)$ \\
No change & $10(33.3 \%)$ \\
Improved & $20(66.7 \%)$ \\
\hline
\end{tabular}

after one month, there was statistically significant improvement in P2 implicit time $(p=0.045)$ and N1P1 amplitude $(\mathrm{p}=0.001)$, there was no statistically significant improvement of N2P2 amplitude ( $\mathrm{p}=0.449)$. In the followup visit after three months for FVEP, there was statistically significant improvement in P2 implicit time $(p=0.002)$, N1P1 amplitude $(\mathrm{p}=0.013)$ and N2P2 amplitude $(p=0.031)$ compared to baseline. Improvement of the
FVEP parameters was recorded in two thirds of the studied cases correlating directly with visual acuity and N2P2 amplitude ratio (Table 5 and 6). According to the findings of FVEP, there was a direct correlation between final visual acuity and initial amplitude of N1p1 and N2P2 and negative correlation with latency of P2 wave. In 20 patients in whom the N1P1 and N2P2 amplitude was within the normal range and amplitude ratio of $\mathrm{N} 2 \mathrm{P} 2$ of normal and fellow eye was at least 0.5 and the P2 implicit time was less than $140 \mathrm{~ms}$, they achieved better visual outcome and visual acuity improved in the affected eye. In the other 10 patients in whom the N1P1 and N2P2 amplitude was below the normal range and the N2P2 amplitude ratio between the normal and the affected eye was less than 0.5 and the P2 implicit time was more than $140 \mathrm{~ms}$, the visual acuity in the affected eye was less than 0.01 and these patients achieved less or no improvement in their visual function. Thus, N2P2 amplitude ratio between the normal and the affected eye show direct correlation with the final visual acuity (Table 7).

Table 4 Comparison Between Normal and Affected According to Flash VEP Including P2 (ms), NIPI ( $\mu \mathrm{v}$ ) and N2P2 ( $\mu \mathrm{v}$ )

\begin{tabular}{|c|c|c|c|c|}
\hline Flash VEP & Normal $(n=30)$ & Affected $(n=30)$ & $\mathbf{t}$ & $\mathbf{p}$ \\
\hline \multicolumn{5}{|l|}{ P2 (ms) } \\
\hline Mean \pm SD & $115.8 \pm 3.4$ & $143.6 \pm 15.6$ & $7.067^{*}$ & $<0.00 I^{*}$ \\
\hline Median (Min. - Max.) & $116(110-120)$ & $145(12|-| 70)$ & & \\
\hline \multicolumn{5}{|l|}{ NIPI $(\mu v)$} \\
\hline Mean \pm SD & $6.1 \pm 1$ & $3.9 \pm 1.9$ & $5.802^{*}$ & $<0.00 I^{*}$ \\
\hline Median (Min. - Max.) & $6(4.5-8)$ & $3.7(I-6)$ & & \\
\hline \multicolumn{5}{|l|}{ N2P2 $(\mu v)$} \\
\hline Mean \pm SD & $17.9 \pm 3.2$ & $7.9 \pm 3.4$ & $7.017^{*}$ & $<0.00 I^{*}$ \\
\hline Median (Min. - Max.) & $17.5(\mid 3-23.5)$ & $9.5(1.6-12)$ & & \\
\hline
\end{tabular}

Note: *Statistically significant at $p \leq 0.05$.

Abbreviations: $t$, paired $t$-test; $p$, $p$ value for comparing between normal and affected. 
Table 5 Comparison Between the Different Periods According to Flash VEP Including P2 (ms), NIPI ( $\mu \mathrm{v}$ ) and N2P2 ( $\mu \mathrm{v}$ )

\begin{tabular}{|c|c|c|c|c|c|}
\hline \multirow[t]{2}{*}{ Flash VEP } & \multirow[t]{2}{*}{ Affected $(n=30)$} & \multicolumn{2}{|c|}{ Follow-Up } & \multirow[t]{2}{*}{$\mathbf{F}$} & \multirow[t]{2}{*}{$\mathbf{p}$} \\
\hline & & One Month $(n=30)$ & Three Months $(n=30)$ & & \\
\hline \multicolumn{6}{|l|}{ P2 (ms) } \\
\hline Mean \pm SD & $143.6 \pm 15.6$ & $138.1 \pm 13$ & $132.5 \pm 12.7$ & $16.465^{*}$ & $<0.00 I^{*}$ \\
\hline Median (Min. - Max.) & $145(|2|-\mid 70)$ & $135(\mid 20-170)$ & $130(|1|-165)$ & & \\
\hline Sig. bet. periods & \multicolumn{3}{|c|}{$\mathrm{P}_{1}=0.045^{*}, \mathrm{P}_{2}=0.002^{*}, \mathrm{P}_{3}<0.00 \mathrm{I}^{*}$} & & \\
\hline \multicolumn{4}{|l|}{ NIPI $(\mu \mathbf{v})$} & & \\
\hline Mean \pm SD. & $3.9 \pm 1.9$ & $4.1 \pm 2$ & $4.2 \pm 2$ & $11.662 *$ & $0.00 I^{*}$ \\
\hline Median (Min. - Max.) & $3.7(I-6)$ & $4(1-6.5)$ & $4(I-6.5)$ & & \\
\hline Sig. bet. periods & \multicolumn{3}{|c|}{$\mathrm{P}_{1}=0.001 *, \mathrm{P}_{2}=0.013^{*}, \mathrm{P}_{3}=1.000$} & & \\
\hline \multicolumn{4}{|l|}{ N2P2 $(\mu \mathrm{v})$} & & \\
\hline Mean \pm SD. & $7.9 \pm 3.4$ & $8.2 \pm 3.2$ & $8.6 \pm 3.3$ & $6.572 *$ & $0.012 *$ \\
\hline Median (Min. - Max.) & $9.5(1.6-12)$ & $9.5(2.3-11.7)$ & $9.5(2.5-12)$ & & \\
\hline Sig. bet. periods & \multicolumn{3}{|c|}{$\mathrm{P}_{1}=0.449, \mathrm{P}_{2}=0.03 \mathrm{I}^{*}, \mathrm{P}_{3}=0.018^{*}$} & & \\
\hline
\end{tabular}

Notes: F: F-test (ANOVA) with repeated measures, Sig. bet. periods was done using post hoc test (adjusted Bonferroni). $\mathrm{p}$ : $\mathrm{p}$ value for comparing between the different periods. $p_{1}: P$ value for comparing between affected and one month follow-up. $p_{2}: p$ value for comparing between affected and three months follow-up. $p_{3}: p_{\text {value for }}$ comparing between one month and three months follow-up. *: Statistically significant at $p \leq 0.05$.

\section{Discussion}

This study aimed to assess the value of the FVEP in determining final visual prognosis in patients with indirect TON. The studied VEP parameters were the $\mathrm{P} 2, \mathrm{~N} 1 \mathrm{P} 1, \mathrm{~N} 2 \mathrm{P} 2$ and N2P2 amplitude ratio between the normal and the affected eye. There was a predominance of males in the studied cases thus paralleling the predominant male prevalence of the condition already reported by other authors. ${ }^{11}$ The age of the studied group was $27.8 \pm 8.74$ paralleling the age reported in other references. ${ }^{12}$

Scrutiny of the clinical data of the study eyes revealed that the initial visual acuity is strong predictor of the final visual outcome moreover those patients who show improvement in visual acuity by the end of the first month continue to improve during the followup period though there was no statistically significant difference between visual acuity by the end of the first month and the third month. As regards FVEP findings, those patients (two third of the studied group) whose $\mathrm{N} 1 \mathrm{P} 1$ and N2P2 amplitude was within the normative data as stated by ISCEV and P2 implicit time less than $140 \mathrm{~ms}$ show significant improvement in the visual acuity. Moreover, those with N2P2 amplitude ratio between the affected and the normal eye was 0.5 or more showed better improvement. The criteria of the studied eyes are in accordance with other published reports. ${ }^{16,17}$ The study of the correlations for the clinical parameters with the VEP parameters reveal a positive correlation between final visual acuity and the initial VEP findings, and between the final visual acuity and the N2P2 amplitude ratio of the normal and the affected eyes both being statistically significant. Thus, N2P2 amplitude ratio between the normal and the affected eye show direct correlation with the final visual acuity.

This study has limitations including the relatively small number of studied eyes, lack of an objective assessment of the optic nerve by OCT for example. Despite the observed diagnostic and predictive role of VEP, it is not easy to place the device to the bedside for patients with multiple injuries. Cases may actually have a concurrent brain injury mistaken for optic nerve damage. ${ }^{17}$ Hence, more studies could avoid these limitations.

FVEP is highly predictive of the final visual outcome in patients having indirect TON provided that the other eye is normal to be used as the patients' internal control. Poor initial visual acuity reduced amplitudes of $\mathrm{N} 1 \mathrm{P} 1$ and N2P2 below the normal range and increased latency of P2 waveform of initial flash VEP and N2P2 ratio between the normal and the affected eye of less than 0.5 indicate poor final visual outcome. Initial better visual acuity, normal N1P1 and N2P2 amplitude indicate a better final visual outcome. 
Table 6 Descriptive Analysis of the Studied Cases According to Improvement of Flash VEP for Each Period Including P2 (ms), NIPI $(\mu v)$ and N2P2 ( $\mu v)$

\begin{tabular}{|c|c|c|}
\hline & & Flash VEP Affected vs: \\
\hline \multirow[t]{8}{*}{ P2 (ms) } & \multicolumn{2}{|c|}{ One month } \\
\hline & Improved & $24(80 \%)$ \\
\hline & No change & $2(6.7 \%)$ \\
\hline & Prolonged & $4(13.3 \%)$ \\
\hline & \multicolumn{2}{|c|}{ Three months } \\
\hline & Improved & $26(86.7 \%)$ \\
\hline & No change & $4(13.3 \%)$ \\
\hline & Prolonged & $0(0 \%)$ \\
\hline \multirow[t]{8}{*}{ NIPI $(\mu v)$} & \multicolumn{2}{|c|}{ One month } \\
\hline & Decreased & $0(0 \%)$ \\
\hline & No change & $6(20 \%)$ \\
\hline & Improved & $24(80 \%)$ \\
\hline & \multicolumn{2}{|c|}{ Three months } \\
\hline & Decreased & $2(6.7 \%)$ \\
\hline & No change & 10 (33.3\%) \\
\hline & Improved & $18(60 \%)$ \\
\hline \multirow[t]{8}{*}{ N2P2 $(\mu v)$} & \multicolumn{2}{|c|}{ One month } \\
\hline & Decreased & $10(33.3 \%)$ \\
\hline & No change & $2(6.7 \%)$ \\
\hline & Improved & $18(60 \%)$ \\
\hline & \multicolumn{2}{|c|}{ Three months } \\
\hline & Decreased & $4(13.3 \%)$ \\
\hline & No change & $4(13.3 \%)$ \\
\hline & Improved & 22 (73.3\%) \\
\hline
\end{tabular}

Table 7 Correlation Between Initial N2P2 Amplitude Ratio and Initial, One Month and Three Months Follow-Up Visual Acuity

\begin{tabular}{|l|c|c|}
\hline \multirow{2}{*}{ Visual Acuity } & \multicolumn{2}{|c|}{ Initial N2P2 Ratio } \\
\cline { 2 - 3 } & $\mathbf{r}_{(\mathbf{s})}$ & $\mathbf{p}$ \\
\hline Initial (baseline) & 0.269 & 0.333 \\
\hline One month (follow-up) & $0.799^{*}$ & $<0.00 I^{*}$ \\
\hline Three months (follow-up) & $0.768^{*}$ & $0.00 I^{*}$ \\
\hline
\end{tabular}

Note: *Statistically significant at $p \leq 0.05$.

Abbreviations: $r$, Pearson coefficient for initial VA; $r_{s}$, Spearman coefficient for VA follow-up.

\section{Abbreviations}

FVEP, flash visual evoked potential; TON, traumatic optic neuropathy; RAPD, relative afferent pupillary defect; CT, computed tomography.

\section{Compliance with Ethical Standards}

- Author contribution: All authors made substantial contributions to conception and design, acquisition of data, or analysis and interpretation of data; took part in drafting the article or revising it critically for important intellectual content; agreed to submit to the current journal; gave final approval of the version to be published; and agree to be accountable for all aspects of the work.

- Data Availability: Available upon request from the corresponding author.

- Informed consent (Ethics): Informed written consent was obtained from of the study participants.

- Consent to Publish (Ethics): Informed written consent was obtained from participants to publish the findings.

Research involving human participants: All procedures performed in studies involving human participants were in accordance with the ethical standards of Alexandria Faculty of Medicine ethics committee and with the 1964 Helsinki declaration and its later amendments. The ethical approval was obtained from the ethics committee of Alexandria Faculty of Medicine, Alexandria, Egypt. A written informed consent was obtained from all individual participants included in the study.

\section{Disclosure}

The authors report no conflicts of interest in this work.

\section{References}

1. Lee V, Ford RL, Xing W, Bunce C, Foot B. Surveillance of traumatic optic neuropathy in the UK. Eye. 2009;24(2):240-250. doi:10.1038/ eye. 2009.79

2. Martinez-Perez R, Albonette-Felicio T, Hardesty DA, Carrau RL, Prevedello DM. Outcome of the surgical decompression for traumatic optic neuropathy: a systematic review and meta-analysis. Neurosurg Rev. 2020;1-9. doi:10.1007/s10143-018-0965-x

3. Steinsapir K, Goldberg R. Traumatic optic neuropathy: a critical update. Compr Ophthalmol Update. 2005;6(1):11-21.

4. Warner N, Eggenberger E. Traumatic optic neuropathy: a review of the current literature. Curr Opin Ophthalmol. 2010;21(6):459-462. doi:10.1097/ICU.0b013e32833f00c9

5. Cirovic S, Bhola RM, Hose DR, et al. Computer modelling study of the mechanism of optic nerve injury in blunt trauma. Br J Ophthalmol. 2006;90(6):778-783. doi:10.1136/bjo.2005.086538

6. Nishi T, Ueda T, Yukawa E, Ohta T, Hara Y. Traumatic optic neuropathy caused by blunt injury to the inferior orbital rim. $J$ Neuro Ophthalmol. 2006;26(1):44-46. doi:10.1097/01. wno.0000205623.83381.6e

7. Singman EL, Daphalapurkar N, White H, et al. Indirect traumatic optic neuropathy. Mili Med Res. 2016;3(1):1-6. doi:10.1186/s40779-0160069-2

8. Walsh FB, Pathological-Clinical Correlations: I. Indirect trauma to the optic nerves and chiasm II. certain cerebral involvements associated with defective blood supply. Invest Ophthalmol Vis Sci. 1966;5 (5):433-449. 
9. Steinsapir KD, Goldberg RA. Traumatic optic neuropathy. Surv Ophthalmol. 1994;38(6):487-518.

10. Steinsapir KD, Goldberg RA. Traumatic optic neuropathy: an evolving understanding. Am J Ophthalmol. 2011;151(6):928-933.e922. doi:10.1016/j.ajo.2011.02.007

11. Holmes MD, Sires BS. Flash visual evoked potentials predict visual outcome in traumatic optic neuropathy. Ophthal Plast Reconstr Surg. 2004;20(5):342-346. doi:10.1097/01.IOP.0000134272.55294.4C

12. Levin LA, Beck RW, Joseph MP, Seiff S, Kraker R. The treatment of traumatic optic neuropathy. Ophthalmology. 1999;106(7):1268-1277. doi:10.1016/S0161-6420(99)00707-1

13. Odom JV, Bach M, Brigell M, et al. ISCEV standard for clinical visual evoked potentials: (2016 update). Doc Ophthalmologica. 2016;133(1):1-9. doi:10.1007/s10633-016-9553-y
14. Robson AG, Nilsson J, Li S, et al. ISCEV guide to visual electrodiagnostic procedures. Doc Ophthalmol. 2018;136(1):1-26. doi:10.1007/s10633-017-9621-y

15. Creel DJ. Visually evoked potentials. Handb Clin Neurol. 2019;160:501-522.

16. Tabatabaei SA, Soleimani M, Alizadeh M, et al. Predictive value of visual evoked potentials, relative afferent pupillary defect, and orbital fractures in patients with traumatic optic neuropathy. Clin Ophthalmol. 2011;5:1021-1026. doi:10.2147/OPTH.S21409

17. Li J, Ran Q, Hao B, Xu X, Yuan H. Transsphenoidal optic canal decompression for traumatic optic neuropathy assisted by a computed tomography image postprocessing technique. $J$ Ophthalmol. 2020;2020:1-6. doi:10.1155/2020/1870745
Clinical Ophthalmology

\section{Publish your work in this journal}

Clinical Ophthalmology is an international, peer-reviewed journal covering all subspecialties within ophthalmology. Key topics include: Optometry; Visual science; Pharmacology and drug therapy in eye diseases; Basic Sciences; Primary and Secondary eye care; Patient Safety and Quality of Care Improvements. This journal is indexed on PubMed

Submit your manuscript here: https://www.dovepress.com/clinical-ophthalmology-journal
Dovepress

Central and CAS, and is the official journal of The Society of Clinical Ophthalmology (SCO). The manuscript management system is completely online and includes a very quick and fair peer-review system, which is all easy to use. Visit http://www.dovepress.com/ testimonials.php to read real quotes from published authors. 